\title{
WILEY-VCH
}

DOI: $10.1002 /(($ please add manuscript number $))$

Article type: Communication

\section{Laser/electron irradiation on InP semiconductor: Promising pathways to in situ formation of In nanoparticles}

Marcelo Assis ${ }^{a}$, Dr. Nadia G. Macedo ${ }^{a}$, Dr. Thales R. Machado ${ }^{a}$, Dr. Mateus. M. Ferrer ${ }^{b}$, Amanda. F. Gouveia ${ }^{a}$, Prof. Dr. Eloisa. Cordoncillo ${ }^{c}$, Dr. Rafael. Torres-Mendieta ${ }^{d}$, Prof. Dr. Hector Beltrán-Mir ${ }^{c}$, Dr. Gladys. Mínguez-Vega ${ }^{e}$, Prof. Dr. Edson. R. Leite ${ }^{a, f}$, Prof. Dr. J. R. Sambrano $^{b}$, Prof. Dr. Juan. Andrés ${ }^{g *}$ and Prof. Dr. Elson. Longo ${ }^{a}$

${ }^{a}$ CDMF, Universidade Federal de São Carlos (UFSCar), P.O. Box 676, CEP, 13565-905 São Carlos-SP, Brazil.

${ }^{\mathrm{b}}$ Modeling and Molecular Simulations Group, São Paulo State University (UNESP), P.O. Box 473, Bauru 17033-360, Brazil.

${ }^{\mathrm{c}}$ Department of Inorganic and Organic Chemistry, University Jaume I (UJI), Castelló 12071, Spain.

${ }^{\mathrm{d}}$ Institute for Nanomaterials, Advanced Technologies and Innovation, Technical University of Liberec, Studentská 1402/2, 46117 Liberec, Czech Republic.

${ }^{\mathrm{e}}$ GROC·UJI, Institut de Noves Tecnologies de la Imatge (INIT, University Jaume I (UJI), Castelló 12071, Spain.

${ }^{\mathrm{f}}$ Brazilian Nanotechnology National Laboratory (LNNano), Brazilian Center for Research in Energy and Materials (CNPEM), Campinas-SP, Brazil.

${ }^{\mathrm{g}}$ Department of Analytical and Physical Chemistry, University Jaume I (UJI), Castelló 12071, Spain.

E-mail: andres@qfa.uji.es

Prof. Juan Andrés*

Keywords: InP, In, femtosecondlaser irradiation, electron beam irradiation, metal nanoparticles

In the current study, we probe whether femtosecond laser and electron beam irradiation of InP are "green," fast, and effective methods to produce metallic In nanoparticles. X-ray diffraction, high-resolution transmission electron microscopy, and energy-dispersive X-ray spectroscopy are employed to investigate the formation and growth of In nanoparticles on InP. Density functional theory and quantum theory of atoms in molecules calculations are 


\section{WILEY-VCH}

employed to reveal the nature of formation of In nanoparticles under electron beam irradiation. These results expand the fundamental understanding of the atomic processes underpinning the mechanism of $\mathrm{In}-\mathrm{P}$ bond rupture during the transformation process induced by the electron irradiation of the InP crystal by increasing the total number of electrons in the bulk structure.

\section{Introduction}

Femtosecond (fs) laser irradiation of solids has played a critical role in materials processing at the micro- and nano-scale. ${ }^{[1,2,3]}$ Moreover, the interactions of an electron beam with matter have been intensively investigated, laying the foundation for transmission electron microscopy (TEM) or scanning electron microscopy (SEM), coupled with various imaging and structural analyses. Such tools provide valuable information on the microstructure, composition, nanoscale properties, and potential applications of the irradiated materials, and the development of techniques to fabricate novel structures. ${ }^{[4]}$ These abundant applications require thorough knowledge of the fundamentals of laser/electron interactions with the target material, for enhanced controllability of the resulting modification of the target relief. Nevertheless, experimental and theoretical methods for investigating these phenomena are limited, and the nature of the mechanisms behind these interactions remains unsolved. Therefore, there is a demand for encouraging the development of new protocols to aid in the design and understanding of the nature of the mechanisms behind these processes.

Significant effort has been devoted to fabricating patterns of metal nanoparticles (NPs) on the surfaces of different substrates owing to their singular properties, which are distinctly different from those of the bulk, with potential use in catalysis, electronic nanodevice development, information storage, etc. ${ }^{[5]}$ In this context, electron beam irradiation inside a 


\section{WILEY-VCH}

TEM or SEM instrument constitutes a direct platform for the formation of metal NPs, whose main advantage is the execution of the mechanisms at the atomic level in nanometric dimensions under conditions of high vacuum at room temperature. It is an excellent methodology for the in situ investigation of nanoscale phenomena, ${ }^{[6]}$ and for the fabrication and manipulation of NPs. ${ }^{[7]}$ The growth processes of several metallic NPs - such as Li, Au, $\mathrm{Cu}, \mathrm{Co},{ }^{[8]}$ and $\mathrm{Ag}$ NPs, which our work group has been focused on — are being studied for technological applications. ${ }^{[9-11,12-16]}$ Accordingly, two in situ synthesis approaches have exhibited promising results - electron beam irradiation in a microscope and fs laser direct irradiation. $^{[2,4]}$

Indium phosphide (InP), like other III-V semiconductors, has a wurtzite or zinc-blende (ZB) crystalline structure, where ZB is the most stable structure at the micrometer scale. ${ }^{[17]}$ The ZB structure belongs to the class of symmorphic space groups and has the $T d$ symmetry as its factor group, with two atoms in the primitive unit cell. ${ }^{[18]}$ The structure of InP is composed of $\left[\mathrm{InP}_{4}\right]$ clusters. However, randomly in the bulk and especially on the surface, there are clusters with undercoordinated In cations with the formation of $\mathrm{P}$ vacancies. The InP material, similar to other compounds of groups III and V, has covalent bonds; however, in this case, owing to the larger electronegativity difference between In and P atoms, the $\mathrm{In}-\mathrm{P}$ bond has a partial ionic character. InP is a semiconductor with a direct band gap energy of $E_{\text {gap }}=$ $1.34 \mathrm{eV}$, low unpassivated surface recombination velocity, ${ }^{[17]}$ and high electron mobility. This band gap is close to the ideal value of the Shockley-Queisser limit in photovoltaics and matches that of the terrestrial solar spectrum, which translates into a theoretical maximum solar conversion efficiency of $31 \%$ (under terrestrial irradiation using a single $\mathrm{p}-\mathrm{n}$ junction). ${ }^{[19]}$ In addition, this semiconductor also satisfies the requirements of an absorber material in a dual-electrode photoelectrochemical water splitting device. ${ }^{[20]}$ InP is also a serious candidate for the production of high-efficiency solar cells ${ }^{[17]}$ and photocathodes for 


\section{WILEY-VCH}

the hydrogen evolution reaction. ${ }^{[21]}$ Other possible applications are its use as a photodetector and laser, and in nanowire development. ${ }^{[22]}$

Golberg et al. ${ }^{[23]}$ pointed out the possibility of the in situ growth of In nanocrystals on the surface of InP nanorods mediated by an electron beam of TEM, for enhancing the optical properties of the semiconductor. According to Gonzalez-Martinez et al. ${ }^{[4]}$, when an irradiated semiconductor meets an electron beam, owing to the laws of energy and impulse, elastic and/or inelastic shocks occur. The electrons that result on inelastic shocks from the collisions with the sample clusters can absorb a fraction of the original electrical energy, with the rest being diverted. The consequences of inelastic shocks include not only scattering of the electrons but also a displacement of the nuclei when the shock surpasses the displacement energy threshold, a value that depends on the chemical composition and atomic structure of the material. The shocks can also lead to the production of secondary or high-energy Auger electrons, thus removing the materials from the equilibrium conditions, resulting in oxidereduction reactions and/or crystallization phenomena.

The irradiation of InP material in vacuum with an electron beam of the TEM induces the weakening of In-P bond and the asymmetric clusters mainly present on the surface start to interact with the electron beam via elastic and inelastic shocks and heating. These defective InP species can be considered as quantum dots, which absorb electrons, causing structural changes owing to polarization effects in the short and medium ranges. The polarization modifies the rotational motion of permanent moments in different $\left[\mathrm{InP}_{4}\right]$ complex clusters, which can cause heating, electrostatic charging, ionization damage (radiolysis), and displacement damage. These phenomena are produced at the atomic level, featuring a quantum phenomenon via the polarization processes on the structural and electronic distorted 


\section{WILEY-VCH}

$\left[\mathrm{InP}_{4}\right]$ clusters by the permanent moment of other neighboring clusters, inducing a symmetry breaking process that promotes the displacement of In from the bulk to the surface. ${ }^{[4]}$

Musaev et al. ${ }^{[24]}$ demonstrated the formation of In NPs on InP wafers via laser-assisted etching and Qian et al. ${ }^{[25]}$ analyzed, for the first time, the evolution of periodic structures on InP (100) surface irradiated with an fs laser. The structural and electronic transformations of the InP material are induced. The absorption of photons in the semiconductor can create random clusters owing to the promotion of a high-density of electron-hole pairs with a large amount of kinetic energy. These "hot" carriers thermalize among each other, promoting the detachment of electrons, holes, and complex symmetric and asymmetric clusters and forming plasma. When the plasma is extinguished, all the hot species experience a coalescence phenomenon stimulated by cooling, thus forming nanoparticles. ${ }^{[2]}$

Our research group is engaged in a project devoted to study of the in situ nucleation and growth processes of Ag NPs on different Ag-based semiconductors and Ag-containing compounds, such as $\alpha-\mathrm{Ag}_{2} \mathrm{WO}_{4},{ }^{[9,26-28]} \beta-\mathrm{Ag}_{2} \mathrm{WO}_{4},{ }^{[12]} \beta-\mathrm{Ag}_{2} \mathrm{MoO}_{4},{ }^{\left[{ }^{[29]}\right.} \beta-\mathrm{AgVO}_{3},{ }^{[15]}$ $\mathrm{Ag}_{2} \mathrm{CrO}_{4},{ }^{[13]}$ and $\mathrm{Ag}_{3} \mathrm{PO}_{4},{ }^{[30]}$ with interesting technological applications such as photoluminescence, microbial agents, and photocatalysts. ${ }^{[9,10,27-29,30,31]}$ In this context, very recently, we have presented the scale-up of the formation of $\mathrm{Ag} \mathrm{NPs}$ on $\alpha-\mathrm{Ag}_{2} \mathrm{WO}_{4}$ with bactericidal properties via fs laser irradiation. ${ }^{[16]}$ We have also recently presented the laserinduced formation of Bi NPs with coexisting crystallographic structures (rhombohedral, monoclinic, and cubic) on $\mathrm{NaBiO}_{3}$ with fundamental focus on laser-based nanofabrication. ${ }^{[32]}$

The objective of the current investigation is threefold. First, we report the formation and growth of In NPs from InP via electron beam irradiation and, to the best of our knowledge, via fs laser-assisted irradiation for the first time. Second, we analyze and compare the modification of the structural properties induced by the electron irradiation and fs laser pulses 


\section{WILEY-VCH}

and establish a correlation between these changes. Third, we aim to apply first-principles calculations, based on density functional theory (DFT) using the quantum theory of atoms in molecules (QTAIM), to determine the structural and electronic properties of InP bulk, and to answer two central questions: (i) What happens when excess electron density, simulating the electron beam of TEM, approaches the surface and bulk of InP? (ii) How are the electrons distributed in this material, and how does this distribution relate to the structural and electronic evolution to form metallic In? We shall discuss how the analysis, provided by both experimental and theoretical results, of the physical and electronic structure of InP allows us to explain the early events in the formation and growth of In. The discussion will address the details of image acquisition and analysis, which will provide a guide to interpret the experimental results. We believe that the present results are of outstanding relevance, as they may inspire efficient synthesis of In NPs, contribute to broadening the fundamental knowledge on the effect of the electron/light-matter interaction, and facilitate an in-depth investigation of the physicochemical behavior and possible applications in various fields (i.e., sensors, catalysis, optical devices, and bio systems).

\section{Results and discussion}

Figure 1 shows the TEM images of an InP sample irradiated with a $200 \mathrm{kV}$ electron beam for $1 \mathrm{~min}$ (Figure 1A), $5 \mathrm{~min}$, (Figure 1B), and $9 \mathrm{~min}$ (Figure 1C). An analysis of the results shows that the InP sample undergoes a change owing to the electron beam irradiation, with the formation of a film of In NPs of different sizes and shapes on the surface. After 5 min of exposure to the beam (Figure 1B), the InP sample becomes saturated with electrons resulting in a diffusion process of In NPs and, consequently, several In NPs and clusters are ejected to the carbon film. Moreover, the number of In NPs attached to the surface of the 


\section{WILEY-VCH}

material is increased, forming a wrap over the irradiated region. Finally, Figure 1C shows that the growth of the film around the surface of the material has stopped, and simultaneously, it shows agglomeration phenomena between the NPs deposited on the carbon film (upper insert in Figure 1B), thus forming larger particles (upper most insert in Figure 1C). It is observed that, as the material is exposed to the electron beam irradiation (Figure 1 (A-C)), the delocalization of In NPs causes the formation of a shell that covers the material. The layer acts as a passivation layer (when a chemical or physical process makes a surface inert) on the material, and consequently, with the progress of time, the material becomes less susceptible to the action of electron irradiation. The elastic shocks from the electron beam irradiation continue to occur, but the inelastic shocks are reduced owing to the formation of a plasmon on the surface, which causes a collective movement of the electrons in the formed metal layer.

A high-resolution TEM (HR-TEM) image of the In NPs deposited on the carbon film and a fast Fourier transform image are shown in the supporting information (Figure S1). An analysis of the results shows the (101), (002), and (110) crystallographic planes, which indicate that the analyzed NPs are metallic tetragonal In, according to the crystallographic card ICSD ( $\left.\mathrm{n}^{\circ} .171679\right),{ }^{[33]}$ belonging to the space group I4/mmm. Thus, we can highlight two main phenomena: the formation of In NPs attached to the InP surface and the release of In NPs with a high crystallinity from the InP matrix.

\section{$<$ Figure 1>}

Figure 1 (D-I) show the on-site HR-TEM images collected with prolonged exposure to electron beam irradiation (inside a TEM of $300 \mathrm{kV}$ electron beam), presenting a better visualization of these phenomena. Initially, in Figure 1D, the indexation of the crystallographic planes of the matrix - (111), (200), (220), and (311) - confirms the presence of cubic InP (crystallographic card JCPDS (N $\mathrm{N}^{\mathrm{o}}$.32-452), space group F-43m). ${ }^{[24]}$ In 


\section{WILEY-VCH}

NPs are formed on the surface of the InP sample with the progress of time; after 4 min of exposure to the beam, (Figure 1E), both the amount and size of In NPs increase (Figure 1F). Figure 1 (G-I) indicate that the In NPs thus formed leave the InP surface and are deposited on the carbon film. In Figure $1(\mathrm{H}-\mathrm{I})$, the index belonging to the metallic tetragonal In planes of both NPs — attached to the surface of the material and deposited in the carbon film — can be identified. By analyzing the images obtained through electron irradiation for 0 to $14 \mathrm{~min}$, the evolution can be summarized as follows: (a) formation of clusters, (b) coalescence of neighboring clusters forming In NPs, (c) displacement of In NPs to the surface, and (d) displacement of In NPs from the surface to further places.

Figure $1(\mathrm{~J}-\mathrm{O})$ present a sequence of HR-TEM images showing the increase in size of tetragonal In NPs with the progress of time of exposure to the electron beam irradiation. Figure $1 \mathrm{~J}$ shows quasi-spherical $\mathrm{In}$ NPs of $\sim 5.6 \mathrm{~nm} \times 5.1 \mathrm{~nm}$ attached to the InP surface, as confirmed by the crystallographic planes index. A visualization of the subsequent figures (Figure $1(\mathrm{~K}-\mathrm{O})$ ) shows the growth process of the In NPs owing to a mass transport from [InP 4 clusters of the InP lattice to the In NPs. These events are accompanied by a morphological deformation, which does not influence the crystallinity of the material.

Figure 2A shows a schematic representation of the 3-D structure of $\mathrm{InP}$ in which the $\left[\mathrm{InP}_{4}\right]$ clusters are highlighted as building blocks of this material. To analyze the effect of the electron beam, calculations were performed by considering the injection of electrons on the InP bulk and analyzing the corresponding response. The calculations begin with the geometrical optimization of the InP structure by increasing the number of electrons of the bulk structure from $N=0$ (neutral) to $N=6 \mathrm{e}$ (charged). Figure $2 \mathrm{~B}$ and $2 \mathrm{C}$ show the evolution of the length bonds of In-P and In-In and the optimized cell parameter as a function of the number of electrons, respectively, where a smooth linear trend is obtained. An analysis of 


\section{WILEY-VCH}

these results shows that the addition of electrons increases the In-P and In-In bond distances, and an expansion of the lattice parameters and cell volume occurs, as shown in Figure 2E. Here, the structural changes induced by the inclusion of electrons into the InP structure can be observed. The In-P bonds are broken and, consequently, the $\left[\mathrm{InP}_{4}\right]$ clusters are structurally and electronically distorted, generating ideal conditions for the growth of In NPs and the formation of defective $\left[\operatorname{In}_{1-\mathrm{x}} \mathrm{P}_{4}\right]$ clusters.

\section{$<$ Figure 2 $>$}

Bader charge densities of each species with the addition of electrons, ${ }^{[34]}$ and the maps of isolines between the In and P elements projected on the (110) plane as a function of electrons added to the unit cell are shown in Figure 2D and 2F, respectively. The electronic charge enclosed within the Bader volume was evaluated for In and P atoms; as the InP contains four atoms of each type, each extra electron is shared among the total eight atoms. An analysis of these results shows that the additional electron imposed in the InP structure is transferred mainly to the In cation. $N$ was selected as $6 e$ per unit cell to represent the necessary number to reduce the In cations to form metallic $\operatorname{In}^{0}$. The calculation results of the charge density, $\rho_{b c p}$, at the $(3,-1)$ bond critical points (BCPs) and the Laplacian of the charge density, $\nabla^{2} \rho_{b c p}$, of In-P bonds in the $\left[\mathrm{InP}_{4}\right]$ clusters are listed in Table 1.

An analysis of these results indicates that the extra electrons affect the atomic charge of both atoms and the neutral atomic configuration of In cation is obtained after adding six electrons to the bulk structure. Under the same conditions, the atomic charge of the $\mathrm{P}$ atom becomes -1.25 . The effect of adding electrons to the material produces differences in the values of the charge density and Laplacian at the $(3,-1)$ BCPs. Thus, the charge density and the Laplacian values of In-P bonds are reduced as the number of added electrons is increased, indicating that these bonds become less strong, which is consistent with the above 


\section{WILEY-VCH}

discussions. Therefore, In cations attract more electrons over the entire irradiation process. Such behavior can be associated with the empty subshells of the outer fifth shell of In atoms (In: $[\mathrm{Kr}] 4 \mathrm{~d}^{10} 5 \mathrm{~s}^{2} 5 \mathrm{p}^{1}$ ), whereas the electron distribution percentage for $\mathrm{P}$ atoms is less as their outer subshell is half-filled ([Ne] $\left.3 \mathrm{~s}^{2} 3 \mathrm{p}^{3}\right)$.

For the InP sample irradiated with the $300 \mathrm{kV}$ electron beam, an increase in the structural and electronic disorder of InP can be observed, as shown in Figure 3. The disorder is caused by the reduction of the In cations, leading to a perturbation of the original crystalline lattice, thus promoting the aggregation of In atoms on the surface of the material. This disorder increases with time of exposure to electron beam irradiation, as shown in Figures 3 (A-F). The purple spheres represent the In cations, expressing mobility with respect to time. Figure $3 \mathrm{G}$ shows that the longer the irradiation time, the more In cations change their position, exhibiting a linear absorption of electronic density, which causes a cumulative structural and electronic disorder in the system. When the disorder in the material increases, In vacancies are generated in the semiconductor as the formal $\mathrm{In}^{3+}$ cation is reduced to metallic $\mathrm{In}^{0}$, and the semiconductor changes from $n$ - to p-type, which leads to a polarization of the crystalline lattice, rendering electron/hole recombination more difficult. Moreover, the material that supports the In NPs is transformed into a non-stoichiometric material, $\operatorname{In}_{1-x} \mathrm{P}$,owing to the absence of In cations inside the crystal structure.

\section{$<$ Figure 3>}

In has a low melting point $\left(156^{\circ} \mathrm{C}\right)$, which renders it a good candidate for the studies of in situ crystallization and recrystallization phenomena. According to Golberg et al., ${ }^{[23]}$ the phenomenon of in situ consecutive rounding and sharpening of In NPs can be attributed to subsequent melting and recrystallization of the material during the growth. Further, it is well known that the $\mathrm{P}$ atom evaporates at low temperatures, and under the electron beam 


\section{WILEY-VCH}

irradiation in TEM conditions, the vapor pressure of $\mathrm{P}$ atom may reach $10^{-6}-10^{-3} \mathrm{~Pa},{ }^{[35]}$ which may induce its detachment out of the semiconductor network and lead to an imbalance between the In cations and $\mathrm{P}$ atoms, and consequently, the remaining In cations bond with other In cations. This process leads to the formation of pure In NPs, with the crystals being detached by the exposure to the electron beam.

The formation and growth processes of In NPs can be considered an adequate method of synthesis induced by electron beam irradiation, where a micrometric precursor is irradiated gradually, experiencing several structural transformations until it suffers an "explosive" reaction, resulting in the ejection of a large number of In NPs, which are deposited in the TEM grid of the carbon film, with the larger particles located closer to the matrix, and the particle size decreasing with the distance from the matrix. Moreover, some In NPs can increase in size gradually with the continuous exposure to the beam. ${ }^{[4]}$ According to Batson et al., ${ }^{[36]}$ electron beam irradiation can induce electromagnetic forces (attractive or repulsive) in groups of nanoscale metal particles, as a plasmonic response to its passage. The nature of these forces depend on the electron beam parameters and the nature of the crystal. ${ }^{[37]}$ Therefore, it is important to consider not only the common interactions between nanoparticles, but also the forces induced by the electron beam irradiation, which can have a scattering effect on the nanoparticles, preventing them from exhibiting complete Brownian motion. ${ }^{[38]}$ Thus, the motion of In NPs induced by the electron beam irradiation can result in a movement of In NPs against each other to generate larger particles, or even in the ejection of particles out of the matrix.

The results obtained with other materials, such as $\mathrm{Ag}_{2} \mathrm{WO}_{4},{ }^{[9-11,12,}{ }^{27]} \mathrm{Ag}_{2} \mathrm{MO}_{4},{ }^{[14]} \beta-$ $\mathrm{AgVO}_{3},{ }^{[15]}$ and $\mathrm{Ag}_{2} \mathrm{CrO}_{4},{ }^{[13]}$ show that specific clusters are capable of absorbing the incoming electrons of the beam, yielding the reduction process from $\mathrm{Ag}^{1+}$ to $\mathrm{Ag}^{0}$. In contrast, the 


\section{WILEY-VCH}

formation and growth processes of In NPs promoted by electron beam irradiation occur in a delocalized manner along the surface of the material. For InP, the above mentioned phenomenon does not occur because the crystal is constituted of only one type of cluster of In, $\left[\mathrm{InP}_{4}\right]$, which is reduced in the entire extension of the irradiated area. As In is a heavy element, it requires higher-energy electrons to be displaced; thus, the interaction with electron beam irradiation is highly dependent on the intensity parameters of the beam. ${ }^{[23]}$ Hence, a conventional SEM system has insufficient power to alter the InP surface. Tetragonal In NPs with defined morphologies (hexagonal and rectangular) were obtained by Bando et al. ${ }^{[23]}$ via electron irradiation inside a TEM at $300 \mathrm{kV}$. However, the In NPs obtained in this study were attached to the surface and no other phenomena, such as their ejection from the surface of InP, were observed.

According to our theoretical model, both In cations and P atoms absorb the electronic charge added, as shown in Figure 2. This behavior is different from that observed in previous works $\left(\mathrm{Ag}_{2} \mathrm{WO}_{4},{ }^{[9-11,12,27]} \mathrm{Ag}_{2} \mathrm{MO}_{4},{ }^{[14]} \mathrm{AgVO}_{3},{ }^{[15]}\right.$ and $\left.\mathrm{Ag}_{2} \mathrm{CrO}_{4}\right) .{ }^{[13]}$ In these materials, $\mathrm{Ag}$ presents a higher ionic characteristic in relation to other species and all absorption of charge by Ag can be compensated by the rearrangement between the different clusters and the generation of displacement defects in the structure. Thus, the Ag almost completely absorbs the charge. However, the covalent nature of the InP material and the existence of only one type of cluster that composes the structure prevent this behavior. As presented here, when InP is irradiated with an electron beam, a series of phenomena is observed: (i) formation of In NPs attached to the InP surface; (ii) formation of a wraparound the original InP network; (iii) segregation of In NPs from the InP surface; (iv) coalescence of In NPs. Therefore, it can be supposed that the superficial In species has a higher probability to reduce to $\operatorname{In}^{0}$, which is step (i). In step (ii), the In metallic NPs can be observed, and subsequent segregation and coalescence of In NPs occurs in step (iii). 


\section{WILEY-VCH}

Figure 4 shows the results of the exposure of InP sample to the fs laser irradiation in ambient environment. Before the irradiation, the morphology of InP is in the form of plaques. After the fs laser irradiation, its morphology is altered and InP spheres surrounded by metal In NPs in the form of islands are observed. An analysis of the crystallographic planes of an isolated sphere reveals that the index of the planes corresponds to tetragonal metallic In (Figure 4B). Although the structure of the metal In NPs remains the same as in the case of the irradiation with TEM, the size and morphologies are different.

\section{$<$ Figure 4 $>$}

The energy-dispersive X-ray spectroscopy (EDS) mapping of the previously fs-laserirradiated InP sample is presented in Figure $4(\mathrm{C}-\mathrm{H})$. Figures $4 \mathrm{C}$ and $4 \mathrm{~F}$ show the dark-field images in the TEM mode of the microscope, illustrating the entire selected analyzed area. Figures 4 (D-E) and (G-H) display the spatially resolved maps of the In and P elements, with an attribution of different colors to each element: In appears on yellow and $\mathrm{P}$ on purple. Therefore, it can be observed that the central spherical particle is composed solely of elementary In with very few traces of $\mathrm{P}$, which are spread over the sample, confirming the results obtained from Figures $4 \mathrm{D}$ and $\mathrm{G}$. The other sections of the image show the original network of InP or the resulting non-stoichiometric $\operatorname{In}_{1-x} \mathrm{P}$ samples.

As in the case of electron beam irradiation, fs laser irradiation is very efficient for the synthesis of In NPs from an InP matrix. However, as mentioned before, the interaction between light and matter involves different phenomena. In this case, the incoming photons can be absorbed by the electrons in the InP sample, promoting a photo-activation process in which the electrons that interact with the incoming photons are either detached out of their respective atomic systems by a multi-photonic absorption process or become simply excited. The electrons located at the structural defects in the lattice require a smaller amount of energy 


\section{WILEY-VCH}

to leave the crystal than that required by the other electrons. One of the ways for the crystal system to recover the equilibrium condition is to segregate its constituent elements. ${ }^{[39]}$

Similar to electron beam irradiation, an fs laser promotes a set of phenomena on the surface of the sample (on the region where the energy deliver is maximized), but in a very short time scale (femtoseconds, picoseconds, and nanoseconds). Therefore, it is possible to promote not only electronic ejection, but also ionic detachment owing to electromagnetic forces, detachment of more electrons and ions owing to collisions between the detached accelerated electrons and the remaining atomic systems, and finally, the formation of an ablation plasma plume containing all the energetic detached species. As mentioned above, the laser-matter interaction leads to the formation of plasma, which continues to interact with the irradiated zone during its lifetime $(\sim \mathrm{ns})$. During the entire interaction, there are fast exchanges of energy while the energetic species move at high velocities owing to an intrinsic acceleration, which results from the ejection process. The movements of the energetic species in the plasma result in an increase in its temperature and pressure. The exposure of the remaining material to the plasma promotes not only more detachment of atoms but also an easier evaporation of $\mathrm{P}$ atoms owing to the pressure and temperature conditions, resulting in the agglomeration of the remaining In atoms. Note that as the process is promoted under ambient conditions, the In atoms could experience an oxidation and reverse oxidation during the lifetime of the plasma. However, after the agglomeration process of In atoms, there is not any evidence of further oxidation.

The surface effects and the type of generated structures (size, morphology) depend on both laser parameters (e.g., pulses and fluences) and the composition of the materials. ${ }^{[24,}$ ${ }^{40]}$ When pulsed laser irradiation is used, the ablation or detachment of material may occur through several mechanisms, such as phase explosion, ${ }^{[41]}$ evaporation, ${ }^{[42]}$ spallation, ${ }^{[43]}$ and 


\section{WILEY-VCH}

fragmentation. ${ }^{[44]}$ After ablation, the sample surface cools at a very high rate and rapidly reorganizes to result in different surface structures. ${ }^{[3,42]}$

As stated before, there are several possible categories of nano/micro structures generated via fs laser irradiation. Figure 4 indicates the presence of irregular nanospheres. We also observed the formation of submicron In spheres detached from the InP matrix and $\operatorname{In}_{x} \mathrm{P}_{y}$ species. Based on the categories of laser-induced structures, it can be concluded that this is a type of protrusion. The formation of irregular In NPs is a consequence of the roughness of the target surface; if the material that absorbs the incoming energy is not being irradiated under the same conditions with the same amount of energy, the detachment of material would not occur in the same way. Hence, the direct and real-time analysis of the formation and growth is performed easily and it facilitates the in-depth understanding of the formation and growth of In NPs.

The interaction of the sample with electron or laser (photons) irradiation leads to polarization of $\left[\mathrm{InP}_{4}\right]$ clusters. At this stage, the crystalline lattice requires a probabilistic description at the atomic level because the crystal symmetry is broken. Thus, structurally disordered $\left[\operatorname{InP}_{4}\right]$ and defective $\left[\operatorname{In}_{1-\mathrm{x}} \mathrm{P}_{4}\right]$ clusters are generated. The movement of these complex clusters in the crystal is highly dependent on the polarization.

In summary, when material processing is induced via irradiation of solids with energetic species such as electrons or photons, it results in structural and atomic defects that affect the common properties of the material. This phenomenon must receive considerable attention owing to its important benefits for the technological applications of the target material. The generation of metallic In NPs is essential to apply these materials in a wide range of technological applications, and in the present work, we exploit the penetrating power of fs laser for the first time, in addition to electron beam irradiation inside a TEM, upon exposing 


\section{WILEY-VCH}

them to InP to obtain In nanoparticles. In both cases, there is an interaction with matter at the quantum level, but the incoming electrons and photons interact differently with the InP sample. This is exemplified in the present work, in which In NPs are obtained via direct fs laser and electron beam irradiation on InP. These processes involve a complex mechanism for the formation, growth, and eventual migration and detachment of In NPs. In the case of electron beam irradiation, the electrons collide inelastically with the electron density cloud of the material, whereas in the case of fs laser irradiation, the photons are absorbed by the complex clusters, inducing, at short and medium distances, modifications in the electron density cloud. This multi-photonic effect results in modifications in the length of In-P bonds, and distortions at the In-P-In angles, owing to perturbations caused by the photons in the crystal structure. The electrons produce a structural modification as a consequence of ordered inelastic shocks, reducing the In cations and forming metallic In NPs and defective $\operatorname{In}_{1-x} P$. When InP is irradiated with an electron beam, a series of phenomena is observed: (i) formation of In NPs attached to the InP surface; (ii) formation of a wraparound the original InP network, and (iii) segregation and coalescence of In NPs at the irradiated InP surface. The photons produce random and disordered structural modifications, owing to the interaction with the different energetic levels of the electronic cloud. In this case, there is an explosive effect, resulting in different species such as In NPs and $\operatorname{In}_{1-x} P$.

\section{Experimental Section}

Synthesis: A commercial reactant indium phosphide (Alfa Aesar, 99.999\% purity) was used to obtain the InP samples used in both synthesis approaches. The route employed to obtain the InP nanostructures via electron beam irradiation is similar to that employed to obtain $\mathrm{Ag}_{2} \mathrm{WO}_{4}$ nanostructures, described in detail by Longo et al. ${ }^{[27]}$. For the synthesis via laser irradiation, InP pellets $(1 \mathrm{~cm} \times 1 \mathrm{~cm})$ were prepared and irradiated under ambient conditions with a Ti: 


\section{WILEY-VCH}

Sapphire laser (Femtopower Compact Pro Femto Lasers), delivering pulses of full width at half maximum of $30 \mathrm{fs}$, with a central wavelength of $800 \mathrm{~nm}$, repetition rate of $1 \mathrm{kHz}$, and maximum average energy per pulse of $0.8 \mathrm{~mJ}$. In order to obtain better pulse compression on the sample, we used an acousto-optic programmable filter (DAZZLER, Faslite). The average power was set to $20 \mathrm{~mW}$ and the beam diameter was set to $6 \mathrm{~mm}$, which was focused on the surface of the target using a $75 \mathrm{~mm}$ lens. The sample was placed on the bottom of a quartz cuvette and attached to a motion-controlled two-dimensional stage, moving at a constant speed of $0.45 \mathrm{~mm} \mathrm{~s}^{-1}$ at the focal plane perpendicular to the laser beam. The irradiation parameter values were selected based on previously reported experiments where the best NP growth in semiconductor networks was reported. ${ }^{[16]}$

Characterization:In situ TEM analysis was performed using a TECNAI G2F20 microscope operating at $200 \mathrm{kV}$ and Titan Themis Cubed (FEI Company, Netherlands) microscopes operating at $300 \mathrm{kV}\left(\mathrm{In}^{0}\right)$. The EDS mapping was performed using a TECNAI G2F20 transmission microscope operating in the scanning mode. The samples were prepared via a simple colloid dropping of the as-prepared InP and laser-irradiated InP on an amorphous carbon film supported on copper grids.

Theoretical Calculations: First-principles calculations were performed within the DFT framework using the VASP program. ${ }^{[45]}$ The Kohn-Sham equations were solved using the Perdew, Burke, and Ernzerhof (PBE) exchange-correlation functional and the electron-ion interaction as described by the projector-augmented-wave pseudo potentials. ${ }^{[46]}$ Owing to the well-known limitations of standard DFT in describing the electronic structure of "strongly correlated" compounds, a correction to the PBE wave functions was adopted (PBE+U) with 


\section{WILEY-VCH}

the inclusion of a repulsive on-site Coulomb interaction $U$, following the method proposed by Dudarev et al. ${ }^{[47]}$ Eventually, the value of the Hubbard parameter was tested and a value of $U$ $=6 \mathrm{eV}$ was used. The plane-wave expansion was truncated at a cut-off energy of $520 \mathrm{eV}$ and the Brillouin zones were sampled through Monkhorst-Pack special $k$-points grids to ensure geometrical and energetic convergence. The initial unit cell parameters and the atomic positions were described according to the ICSD crystallographic information file (CIF) $n^{\circ}$ 640186. ${ }^{[48]}$ The keyword NELECT was used to increase the number of electrons in the bulk structure, and all the crystal structures were optimized. The relationship between the charge density topology and elements of molecular structure and bonding was noted by Bader. ${ }^{[49]}$ This relationship, Bader's QTAIM, ${ }^{[49,50]}$ is a well-recognized tool used to analyze electron density, describe interatomic interactions, and rationalize chemical bonding. The different strong and weak interactions between two atoms can be determined unequivocally using QTAIM calculations. According to the standard QTAIM framework, concepts such as $(3,-1)$ BCPs, their respective bond paths, and $L(r)=-\nabla^{2} \rho(r)$ maps can be analyzed to reveal the nature of these interactions.

\section{Supporting Information}

Supporting Information is available from the Wiley Online Library or from the author.

\section{Acknowledgments}

The authors acknowledge the financial support of agencies: CAPES/PNPD, FAPESP (2013/07296-2; 2013/26671-9), CNPq (150250/2017-1, 166281/2017-4), Generalitat Valenciana for PrometeoII/2014/022, Prometeo/2016/079, AICO/2016/036, 


\section{WILEY-VCH}

ACOMP/2014/270, ACOMP/2015/1202, Ministerio de Economia y Competitividad, project CTQ2015-65207-P, FIS2016-75618-R and MAT2016-80410-P, and Programa de Cooperación Cientifica con Iberoamerica (Brasil) of Ministerio de Educación (PHBP1400020) and R. T-M acknowledges the Ministry of Education, Youth and Sports of the Czech Republic for financial support through the projects No. LM2015073 and LO1201. J.A. acknowledges Ministerio de Economia y Competitividad, "Salvador Madariaga" program, PRX15/00261. E.C. and H.B.M. thank the "Universitat Jaume I" project N". UJIB2016-38, andJ.A. acknowledges “Universitat Jaume I" project No. UJI-B2016-25 for financial support. M.J. also expresses gratitude to this project for a fellowship. The authors are very grateful to the "Serveis Centrals d'Instrumentació Científica (SCIC)" of the University Jaume I for the use of the femtosecond laser equipment and, to the "Brazilian Nanotechnology National Laboratory - LNNano" for the use of the microscope facilities. The authors would especially like to express gratitude to Santiago Maya-Johnson and Rori Camargo for their stimulating discussions and help in TEM operation.

Received: ((will be filled in by the editorial staff))

Revised: ((will be filled in by the editorial staff))

Published online: ((will be filled in by the editorial staff))

\section{References}

[1] a) F. Korte, J. Koch, A. Egbert, C. Fallnich, A. Ostendorf, B. N. Chichkov, Appl. Phys. A 2003, 77, 229; b) M. Haque, K. K. Lee, S. Ho, L. A. Fernandes, P. R. Herman, Lab Chip 2014, 14, 3817; c) K.-S. Lee, R. H. Kim, D.-Y. Yang, S. H. Park, Prog. Polym. Sci. 2008, 33, 631; d) K. K. Seet, V. Mizeiks, S. Matsuo, S. Juodkazis,H. Misawa, Adv. Mater. 2005, 17, 541; e) M. Malinauskas, A. Žukauskas, S. Hasegawa, Y. Hayasaki, V. Mizeikis, R. Buividas, S. Juodkazis, Light: Sci. Appl. 2016, 5, e16133; f) L. Wang, Q. Li, H.-Y. Wang, J.C. Huang, R. Zhang, Q.-D. Chen, H.-L. Xu, W. Han, Z.-Z. Shao, H.-B. Sun, Light: Sci. Appl.2015, 4, e245; g) S. S. Chou, B. S. Swartzentruber, M. T. Janish, M, K. C.Meyer, L. B. Biedermann, S. Okur, D. B. Burckel, C. B. Carter, B. Kaehr, Appl. Phys. A 2016, 7, 3736; h) G. D. Tsibidis, E. Skoulas, A. Papadopoulos, E. Stratakis, Phys. Rev. B 2016, 94. 


\section{WILEY-VCH}

[2] W. Xiong, Y. Zhou, W. Hou, L. Jiang, M. Mahjouri-Samani, J. Park, X. He, Y. Gao, L. Fan, T. Baldacchini, J.-F. Silvain, Y. Lu, Front. Optoelectron. 2015, 8, 351.

[3] A. Y. Vorobyev, C. Guo, Laser Photonics Rev. 2012, 7, 385.

[4] I. G. B. Gonzalez-Martinez, A. Bachmatiuk, V. Bezugly, J. Kunstmann, T. Gemming, Z. Liu, G. Cuniberti, M. H. Rümelli, Nanoscale 2016, 08, 1.

[5] a) G. A. Ozin, A. Arenault, L. Cademartiri, Nanochemistry: A Chemical Approach to Nanomaterials: Edition 2, RCS Publishing, Cambridge, U.K. 2009; b) M. Cargnello, W. DoanNguyen, T. R. Gordon, R. E. Diaz, E. A. Stach, R. J. Gorte, P. Fornasiero, C. B. Murray, Science 2013, 341, 771; c) J. Cardellino, N. Scozzaro, M. Herman, A. J. Berger, C. Zhang, K. C. Fong, C. Jayaprakash, D. V. Pelekhov, P. C. Hammel, Nat. Nanotechnol. 2014, 9, 343; d)P. Wu, X. P. Yan, Chem. Soc. Rev. 2013, 42, 5489.

[6] a) T. L. Ben-David, Y. Lereah, G. Deutscher, J. M. Penisson, A. Bourret, R. Kofman, P. Cheyssac, Phys. Rev. Lett. 1997, 78, 2585; b) R. Wang, H. Zhang, M. Farle, C.

Kisielowski, Nanoscale 2009, 1, 276.

[7] a) M.-Y. Yen, C.-W. Chiu, F.-R. Chen, J.-J.Kai,C.-Y. Lee, H.-T. Chiu, Langmuir 2004, 20, 279; b) D. Tham, C. Y. Nam, J. E. Fischer, Adv. Mater. 2006, 18, 290.

[8] a) J. Do, M. Fedoruk, F. Jackel, J. Feldmann, Nano Lett. 2013, 13, 4164; b) M. A. Huergo, C. M. Maier, M. F. Castez, C. Vericat, S. Nedev, R. C. Salvarezza, A. S. Urban, J. Feldmann, ACS Nano 2016, 10, 3614; c) M. J. Guffey, R. L. Miller, S. K. Gray, N. F. Scherer, Nano Lett. 2011, 11, 4058.

[9] W. d. S. Pereira, J. Andrés, L. Gracia, M. A. San-Miguel, E. Z. da Silva, E. Longo, V. M. Longo, Phys. Chem. Chem. Phys. 2015, 17, 5352.

[10] E. Longo, D. P. Volanti, V. M. Longo, L. Gracia, I. C. Nogueira, M. A. P. Almeida, A. N. Pinheiro, M. M. Ferrer, L. S. Cavalcante, J. Andrés, J. Phys. Chem. C 2014, 118, 1229.

[11] a) M. A. San-Miguel, E. Z. da Silva, S. M. Zannetti, M. Cilense, M. T. Fabbro, L. Gracia, J. Andres, E. Longo, Nanotechnol. 2016, 27, 225703; b) E. Longo, W. Avansi, Jr., J. Bettini, J. Andres, L. Gracia, Sci. Rep. 2016, 6, 21498.

[12] R. A. Roca, A. F. Gouveia, P. S. Lemos, L. Gracia, J. Andrés, E. Longo, Inorg. Chem. $2016,55,8661$.

[13] M. T. Fabbro, L. Gracia, G. S. Silva, L. P. S. Santos, J. Andrés, E. Cordoncillo, E. Longo, J.Solid State Chem. 2016, 239, 220.

[14] M. T. Fabbro, C. Saliby, L. R. Rios, F. A. La Porta, L. Gracia, M. S. Li, J. Andres, L. P. Santos, E. Longo, Sci. Technol. Adv. Mat. 2015, 16, 065002.

[15] R. C. de Oliveira, M. Assis, M. M. Teixeira, M. D. P. da Silva, M. S. Li, J. Andres, L. Gracia, E. Longo, J. Phys. Chem. C 2016, 120, 12254. 


\section{WILEY-VCH}

[16] M. Assis, E. Cordoncillo, R. Torres-Mendieta, H. Beltran-Mir, G. Minguez-Vega, R. Oliveira, E. R. Leite, C. C. Foggi, C. E. Vergani, E. Longo, J. Andres, Sci. Rep. 2018, 8, 1884.

[17] J. A. Wallentin, N. Anttu, D. Asoli, M. I. Aberg, M. H. Magnusson, G. Siefer, P. FussKailuweit, F. Dimroth, B. Witzigmann, H. Q. Xu, L. Samuelson, K. Deppert, M.T. Borgström, Sciencexpress 2013, 1. 1230969.

[18] P. E. Faria Junior, G. M. Sipahi, J. Appl. Phys. 2012, 112, 103716.

[19] C. H. Henry, J. Appl. Phys. 1980, 51, 4494.

[20] L. C. Seitz, Z. Chen, A. J. Forman, B. A. Pinaud, J. D. Benck, T. F. Jaramillo, ChemSusChem 2014, 7, 1372.

[21] L. Gao, Y. Cui, J. Wang, A. Cavalli, A. Standing, T. T. Vu, M. A. Verheijen, J. E. Haverkort, E. P. Bakkers, P. H. Notten, Nano Lett. 2014, 14, 3715.

[22] a) M. Yoshimura, E. Nakai, K. Tomioka, T. Fukui, Appl. Phys. Express 2013, 6, 052301; b) J. Wallentin, N. Anttu, D. Asoli, M. Huffman, Aberg, I.; M. H. Magnusson, G. Siefer, P. Fuss-Kailuweit, F. Dimroth, B. Witzigmann, H. Q. Xu, L. Samuelson, K. Deppert, M. T. Borgström, Sciencexpress 2013, 1230969; c)Z. Wang, B. Tian, M. Paladugu, M. Pantouvaki, N. Le Thomas, C. Merckling, W. Guo, J. Dekoster, J. Van Campenhout, P. Absil, D. Van Thourhout, Nano Lett. 2013, 13, 5063; Y. Yu, C. Yu, T. Xu, H. Sun, Mater. Sci. Semicond. Process. 2017, 68, 270.

[23] D. Golberg, M. Mitome, L. W. Yin, Y. Bando, Chem. Phys. Lett. 2005, 416, 321.

[24] O. R. Musaev, V. Dusevich, J. M. Wrobel, M. B. Kruger, J. Mater. Sci. 2011, 46, 3157 .

[25] H. X. Qian, W. Zhou, H. Y. Zheng, X. R. Zeng, H. C. Sheng, Mater. Lett. 2014, 124, 235.

[26] J. Andres, L. Gracia, P. Gonzalez-Navarrete, V. M. Longo, W. Avansi, Jr., D. P. Volanti, M. M. Ferrer, P. S. Lemos, F. A. La Porta, A. C. Hernandes, E. Longo, Sci.Rep. 2014, 4, 5391.

[27] E. Longo, L. S. Cavalcante, D. P. Volanti, A. F. Gouveia, V. M. Longo, J. A. Varela, M. O. Orlandi, J. Andres, Sci. Rep. 2013, 3, 1676.

[28] J. Andres, L. Gracia, A. F. Gouveia, M. M. Ferrer, E. Longo, Nanotechnol. 2015, 26, 405703.

[29] a) Y. V. B. De Santana, J. E. C. Gomes, L. Matos, G. H. Cruvinel, A. Perrin, C. Perrin, J. Andrès, J. A. Varela, E. Longo, Nanomater. Nanotechnol. 2014, 4, 22; b) J. Andrés, M. M. Ferrer, L. Gracia, A. Beltran, V. M. Longo, G. H. Cruvinel, R. L. Tranquilin, E. Longo, Part. Part.Syst. Charact. 2015, 32, 646.

[30] G. Botelho, J. Andres, L. Gracia, L. S. Matos, E. Longo, ChemPlusChem 2016, 81, 202. 


\section{WILEY-VCH}

[31] a) Z. L. Lin, J.; Zheng, Z.; Yan, J.; Liu, P.; Wang, C.; Yang, G., ACS Nano 2015, 9, 7256; b) Z. Y. Bao, D. Y. Lei, J. Dai, Y. Wu, Appl. Surf. Sci. 2013, 287, 404; c) J. Andrés, A. F. Gouveia, L. Gracia, E. Longo, G. Manzeppi Faccin, E. Z. da Silva, D. H. Pereira, M. A. San-Miguel, Int. J. Quantum Chem. 2017, e25551.

[32] M. Assis, E. Cordoncillo, R. Torres-Mendieta, H. Beltrán-Mir, G. Mínguez-Vega, A. G. Fernandes, E. R. Leite, J. Andrés, E. Longo, Phys. Chem. Chem. Phys. 2018, $10.1039 / \mathrm{C} 8 \mathrm{CP} 01225 \mathrm{C}$.

[33] E. G. Moshopoulou, R. M. Ibberson, J. L. Sarrao, J. D. Thompson, Z. Fisk, Acta Cryst. 2006, B62, 173.

[34] a) R. F. W. Bader, Acc. Chem. Res. 1985, 18, 9; b) R. F. W. Bader, Chem. Rev. 1991, $91,893$.

[35] G. V. Samsonov, Handbook of Physicochemical Properties of the Elements. IFI/Plenum Data Corporation, 1968.

[36] P. E. Batson, A. Reyes-Coronado, R. G. Barrera, A. Rivacoba, P. M. Echenique, J. Aizpurua, Nano Lett. 2011, 11, 3388.

[37] H. G. Liao, K. Niu, H. Zheng, Chem. Comm. 2013, 49, 11720.

[38] H. Zheng, U. M. Mirsaidov, L. W. Wang, P. Matsudaira, Nano Lett. 2012, 12, 5644.

[39] a) A. Kumar, S. Rajauria, H. Huo, O. Ozsun, K. Rykaczewski, J. Kumar, K. L. Ekinci, Appl. Phys. Lett. 2012, 100, 141607; G. F. b) G. F. Walsh, L. Dal Negro, Nanoscale 2013, 5, 7795; c) S. Höhm, A. Rosenfeld, J. Krüger, J. Bonse, Opt. Express 2015, 23, 25959.

[40] G. Baraldi, J. Gonzalo, J. Solis, J. Siegel, Nanotechnol. 2013, 24, 255301.

[41] N. M. Bulgakova, I. M. Burakov, Appl. Surf. Sci. 2002, 197-198, 41; R. Eason, Wiley 2007.

[42] S. I. L. I. Anisimov, B. S. Luk'Ianchuk, Phys.-Usp. 2002, 45, 293.

[43] N. A. Inogamov, V. V. Zhakhovsky, V. A. Khokhlov, S. I. Ashitkov, Y. N. Emirov, K. V. Khichshenko, A. Y. Faenov, T. A. Pikuz, M. Ishino, M. Kando, N. Hasegawa, M. Nishikino, P. S. Komarov, B. J. Demaske, M. B. Agranat, S. I. Anisimov, T. Kawachi, I. I. Oleynik, J.Phys.: Conf. Ser. 2014, 510, 012041.

[44] D. Perez, L. J. Lewis, Phys. Rev. Let. 2002, 89, 255504.

[45] a) G. Kresse, J. Frurthmüller, Phys. Rev. B 1996, 54, 11169 ; G. b) Kresse, J. Frurthmüller, Comp. Mater. Sci. 1996, 6, 15.

[46] J. P. Perdew, K.Burke, M. Ernzerhof, Phys. Rev. Lett. 1996, 77, 3865; G. J. Kresse, D. Joubert, Phys. Rev. B 1999, 59, 1759.

[47] S. L. Dudarev, G. A. Botton, S. Y. Savrasov, C. J. Humphreys, A. P. Sutton, Phys. Rev. B 1998, 57, 1505. 


\section{WILEY-VCH}

[48] A. G. Thompson, J. E. Rowe, M. Rubenstein, J. Appl. Phys. 1969, 40, 3280.

[49] R. F. W. Bader, Atoms in Molecules: a Quantum Theory. Claredon Press, Oxford 1990.

[50] C. F. Matta, R. J. Boyd, The Quantum Theory of Atoms in Molecules: from Solid State to DNA and Drug Design. Wiley-VCH, Weinheim, Germany 2007.
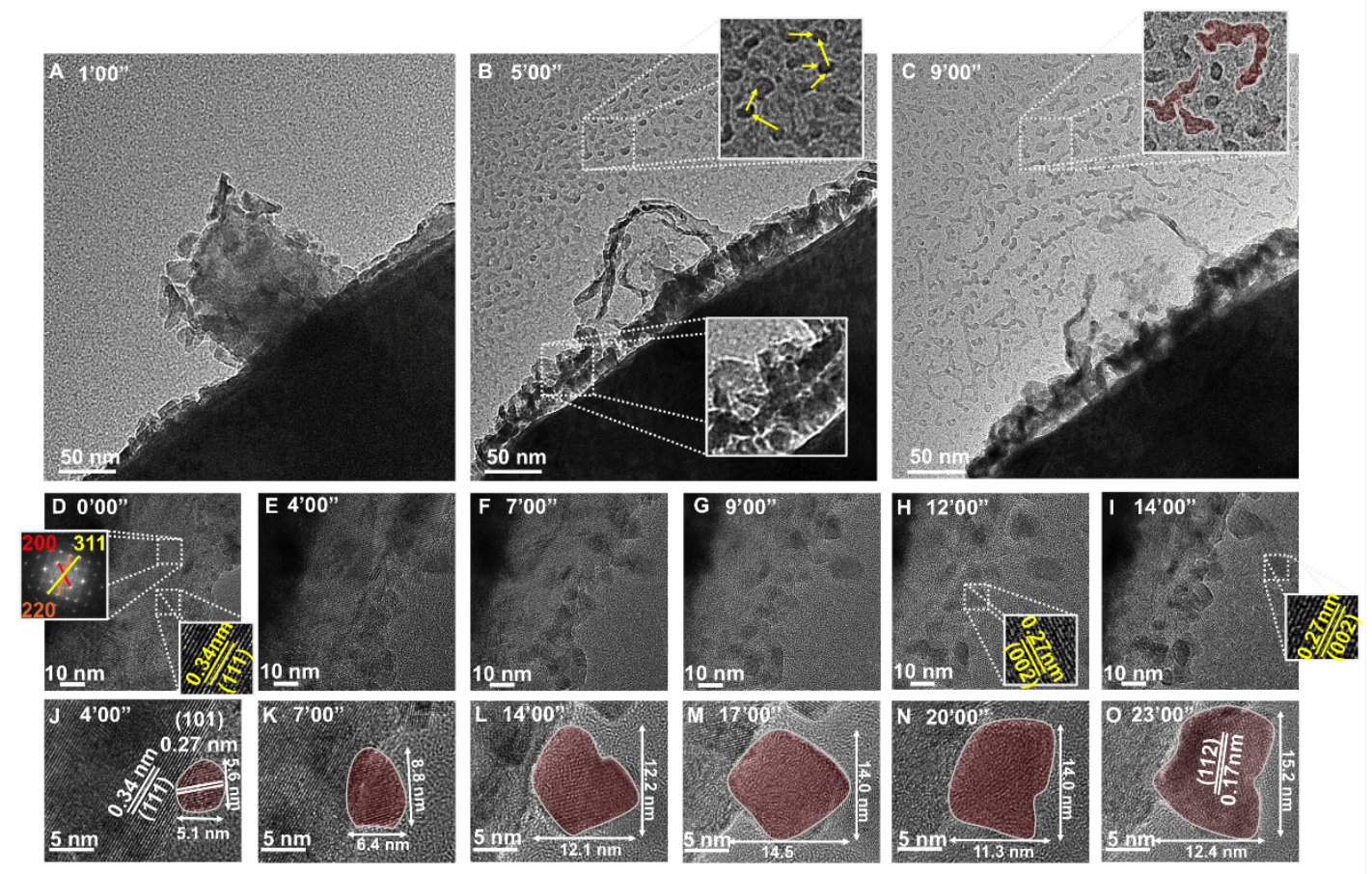

Figure 1. TEM images of InP sample irradiated by electron beam at different times: (A) 1 min; formation of In NP on InP surface; (B) $3 \mathrm{~min}$; -decrease in thin film growth and InP nanoparticle blast with formation of nanoparticles and clusters on the carbon screen and (C) 5 min-stop the growth of the film around InP and random distribution of NPs and clusters. The insets show magnified images of the pointed regions. (D-I): Sequence of in situ HR-TEM images showing the growth and detachment of metallic Indium nanoparticles from the InP structure within the time of exposure to an electron beam. (J-O) Time resolved HR-TEM images of an In NP on the InP surface. 
WILEY-VCH

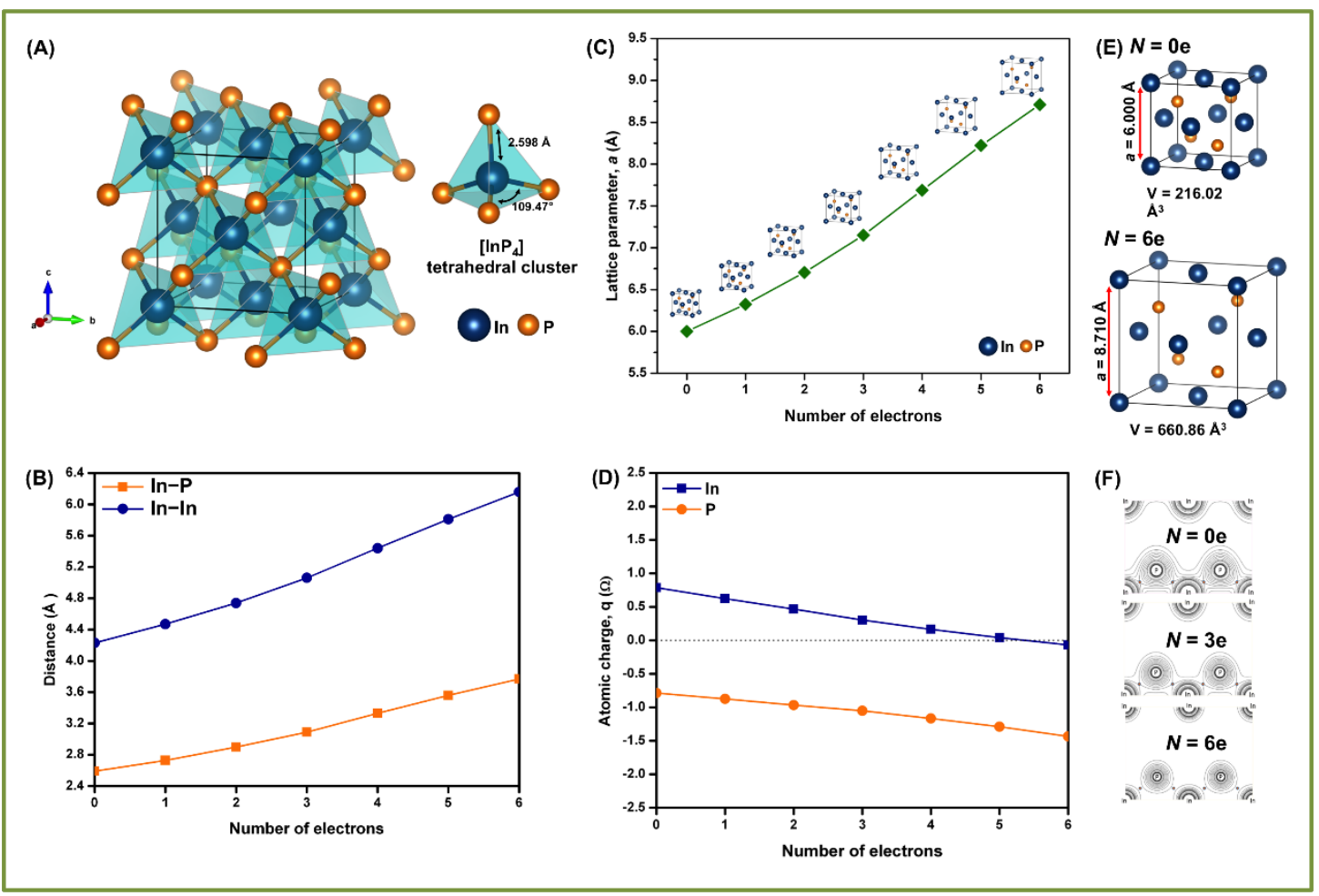

Figure 2. (A) 3-D structure of $\mathrm{InP}$ composed by tetrahedral $\left[\mathrm{InP}_{4}\right]$ clusters. (B) Bonds distances of In-P and In-In, (C) Lattice parameter ( $a$ ), and (D) Atomic charge ( $q$ ) as function of number of electrons. (E) Cell volume when $N=0 \mathrm{e}$ and $N=6 \mathrm{e}$. (F) 2-D schematic representation of the Barder charges projected on the (110) surface of the InP crystal.
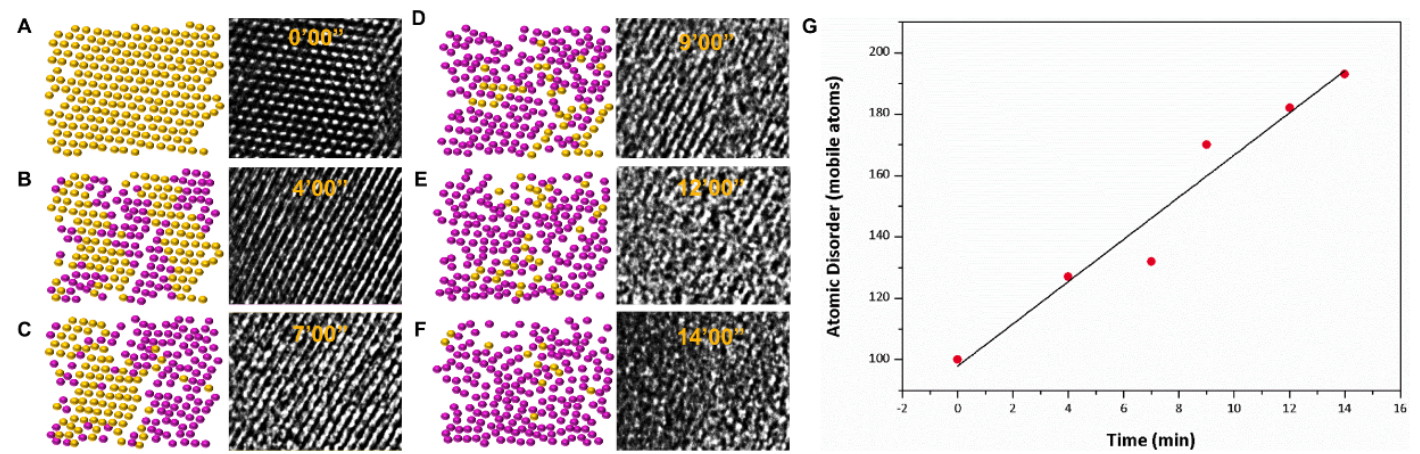

Figure 3. (A-F) HR-TEM images of InP showing the scheme to structural disorder. (G) Evolution of atomic disorder with time. 


\section{WILEY-VCH}
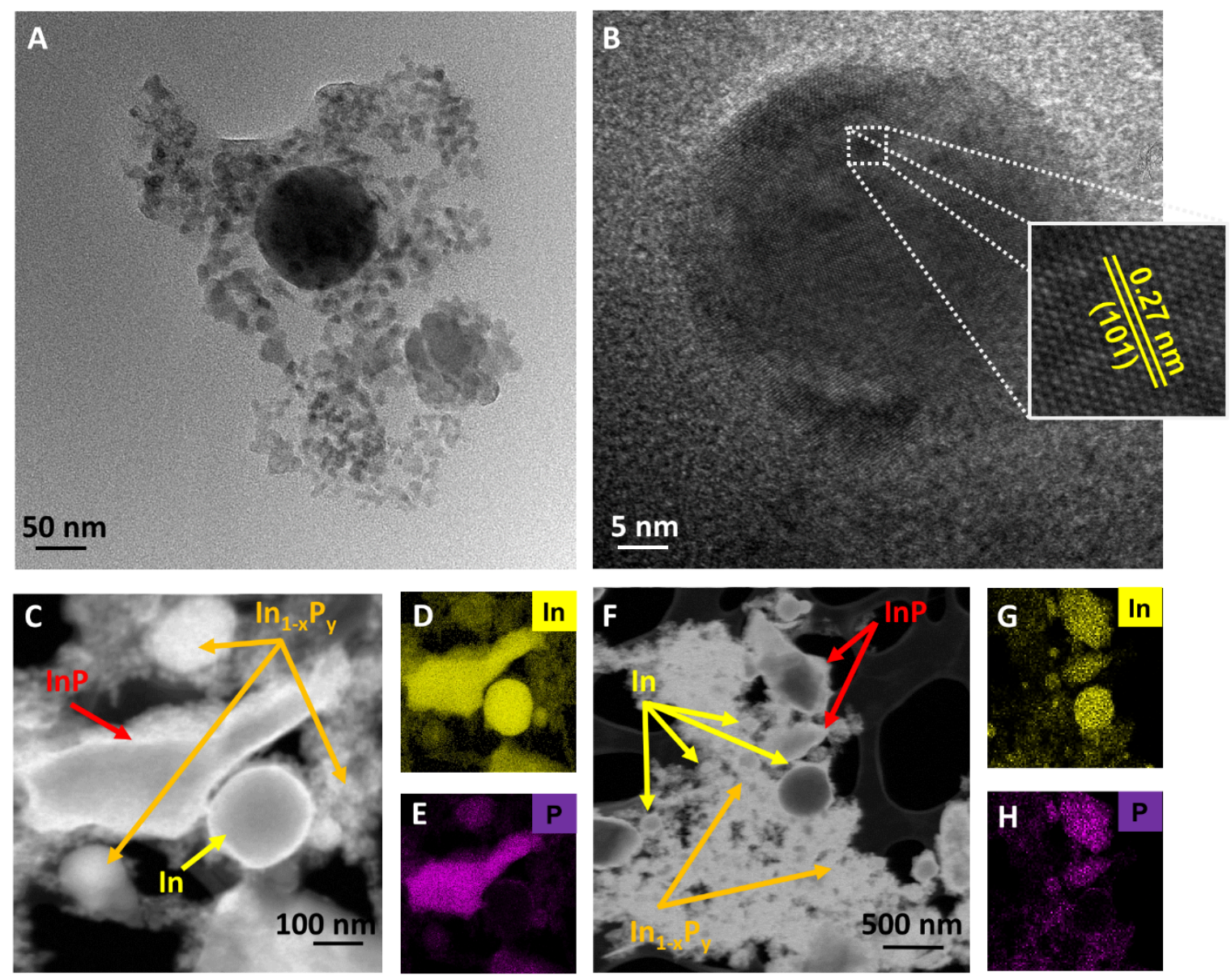

Figure 4. (A) TEM image of the InP sample after the exposure to the fs laser irradiation. (B) HR-TEM image of the In particle and Inset showing its indexed interplanar distances. (C-H) Energy-dispersive X-ray spectroscopy (EDS) mapping of InP sample irradiated by fs laser.

Table 1. QTAIM results of the charge density, $\rho_{b c p}$, at the $(3,-1)$ bond critical points $(\mathrm{BCP})$ as well as its Laplacian, $\nabla^{2} \rho_{\text {bcp }}$, in In-P.

\begin{tabular}{cccccccc}
\hline Number of electron & $\mathbf{0}$ & $\mathbf{1}$ & $\mathbf{2}$ & $\mathbf{3}$ & $\mathbf{4}$ & $\mathbf{5}$ & $\mathbf{6}$ \\
\hline $\boldsymbol{\rho}_{b c p}$ & 0.40 & 0.33 & 0.25 & 0.19 & 0.14 & 0.10 & 0.08 \\
$\boldsymbol{\nabla}^{2} \boldsymbol{\rho}_{b c p}$ & 1.125 & 1.000 & 0.740 & 0.530 & 0.390 & 0.310 & 0.280 \\
& & & & & & & \\
\hline
\end{tabular}




\section{WILEY-VCH}

In this communication, we present two efficient and green methods for the formation of metallic In nanoparticles on the surface of InP semiconductor material. Combining the in situ study of the NPs formation with first-principles calculations, we are able to explain, at quantum level, the events involved on the In NP growth, both by the electrons and photons participation.

Keywords: InP, In, femtosecondlaser irradiation, electron beam irradiation, metal nanoparticles

Marcelo Assis $^{a}$, Dr. Nadia G. Macedo ${ }^{a}$, Dr. Thales R. Machado ${ }^{a}$, Dr. Mateus. M. Ferrer ${ }^{b}$, Amanda. F. Gouveia ${ }^{a}$, Prof. Dr. Eloisa. Cordoncillo ${ }^{c}$, Dr. Rafael. Torres-Mendieta ${ }^{d}$, Prof. Dr. Hector Beltrán-Mirc, Dr. Gladys. Mínguez-Vega ${ }^{e}$, Prof. Dr. Edson. R. Leite ${ }^{a, f}$, Prof. Dr. J. R. Sambrano $^{b}$, Prof. Dr. Juan. Andrés ${ }^{g^{*}}$ and Prof. Dr. Elson. Longo ${ }^{a}$

Laser/electron irradiation on InP semiconductor: A promising pathway to in situ formation of In nanoparticles

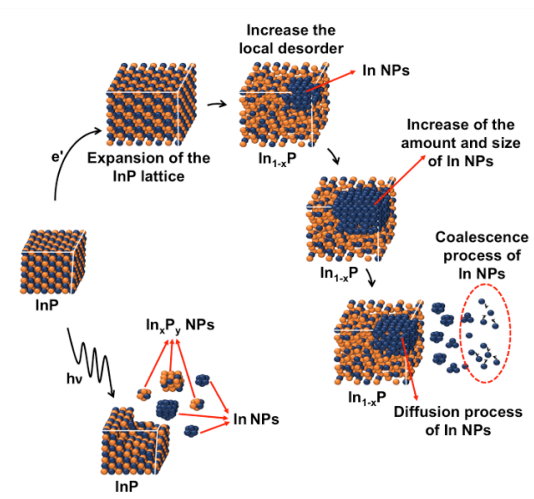

Supporting Information

Laser/electron irradiation on InP semiconductor: A promising pathway to in situ formation of In nanoparticles 


\section{WILEY-VCH}

Marcelo Assis $^{a}$, Dr. Nadia G. Macedo ${ }^{a}$, Dr. Thales R. Machado ${ }^{a}$, Dr. Mateus. M. Ferrer ${ }^{b}$, Amanda. F. Gouveia ${ }^{a}$, Prof. Dr. Eloisa. Cordoncillo ${ }^{c}$, Dr. Rafael. Torres-Mendieta ${ }^{d}$, Prof. Dr. Hector Beltrán-Mirc, Dr. Gladys. Mínguez-Vega e, Prof. Dr. Edson. R. Leite ${ }^{a, f}$, Prof. Dr. J. R. Sambrano $^{b}$, Prof. Dr. Juan. Andrés ${ }^{g^{*}}$ and Prof. Dr. Elson. Longo ${ }^{a}$

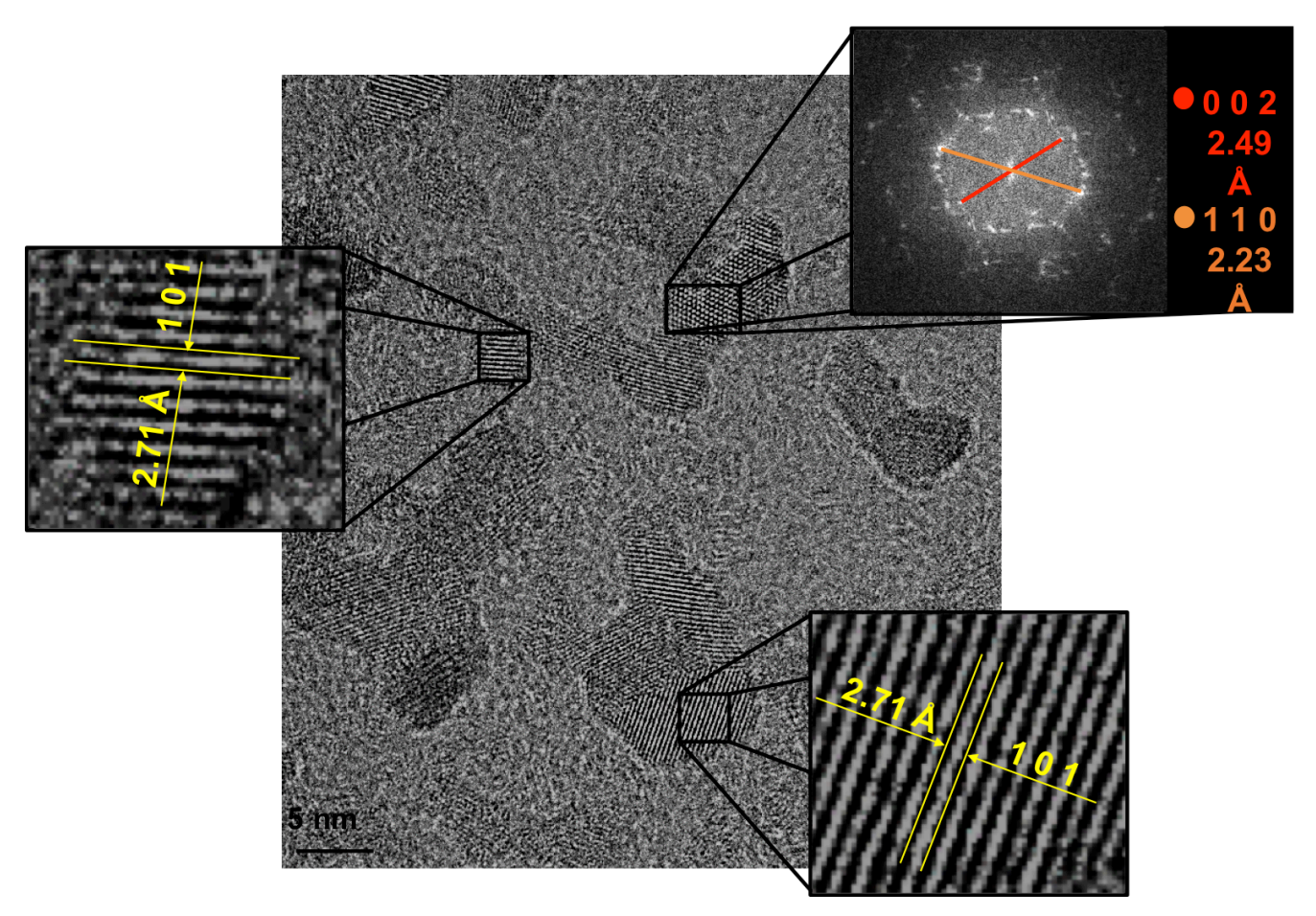

Figure S1: High-resolution TEM (HR-TEM) image and fast Fourier transform image of the In NPs deposited on carbon film. 